\title{
Finite element modeling of a moving load using contact conditions
}

\author{
Sergey Gridnev, ${ }^{1, *}$, and Ilya Ravodin ${ }^{1}$ \\ ${ }^{1}$ VSTU, Structural Mechanics Department, 394026, Voronezh, Russia
}

\begin{abstract}
The article deals with the issues of simulation of the dynamic moving load on a span structure in the modern finite element systems. The possibility of using the contact conditions in the Midas NFX program to describe the coupled vibrations of a beam and a movable load is investigated. To verify the results obtained, the method of separate modeling of the moving load by use of several forces on the trafficway line is used. The series of numerical studies of an elastically supported dynamical system was performed using two techniques. Graphic charts are obtained mapping vertical displacements of the vibration system characteristic points, depending on the inertial properties of the moving load, on the nodal forces application step, the parameters of surface conditions.
\end{abstract}

\section{Introduction}

In the design of road bridges with different bearing systems, the dynamic effects of vehicles must be taken into account. The forces of inertia of a moving car and the bridge span structure in interaction with it in most cases have a significant impact on the nature of joint oscillations.

One of the directions of studying the features of the dynamic impact of the moving load on the bridge structures in modern conditions is the use of modern software systems that implement the finite element method (FEM). The development of new or improvement of existing algorithms for mobile load modeling allows to specify dynamic coefficients and to make rational engineering decisions in the design of new and evaluation of the technical condition of existing transport facilities.

Let us focus on the recently used fundamental approaches to solving problems of modeling the impact of mobile load. The DynSys software package (in $\mathrm{C}++$ programming language) was developed in the paper [1] on the basis of MSC/Nastran universal software solution for finite element analysis to calculate the load and span structure vibration. DynSys implements an iterative method of solving equations of the motion of the dynamic complex "bridge-moving load". It is considered that the mobile load experiences a dynamic impact from the bridge, moving along an uneven path - the contact lines. In its turn, the interaction of the moving load on the bridge is described by a group of variables in terms of magnitude and position of the nodal loads. Thus, a step-by-step calculation of two dynamic

\footnotetext{
* Corresponding author: gridnev_s_y@,rambler.ru
} 
systems is carried out: moving load and span structure. In this case, the connecting link is the displacement of the contact lines.

In the article [2] the possibility of mixed simulation of railway bridge and train vibrations is considered, on the basis of the "Universal Mechanism" software package. The finite element design model is represented by a span structure and a moving trolley model that consists of a set of absolutely rigid bodies connected by means of hinges and power elements. The interaction between the wheel and the rail is described using the simplified theory of rolling contact of J. Kalker. The forces of interaction between the wheel and the rail are calculated at each time step of the numerical solution. This approach allows us to determine with high accuracy the "response" forces acting on the part of the span structure on the dynamic model of the train, but it is applicable only for modeling railway transport.

In the paper [3], for modeling of coupled vibrations of a span structure and a mobile load, the author uses a finite element computer program developed at the "Construction mechanics" Department of MIIT [4]. As in [2], the connection between the mobile load and the span structure is established through the forces of contact interaction; however, the movement of the finite-element model of the crew occurs due to the sliding of its support nodes along the contact surface. It is assumed that at the interface between the moving load and the bridge there are forces that prevent interpenetration, which are given by the rule of the shoulder at the nodes. The reaction vector resolves into a normal and a tangential component to the contact boundary. To determine the reactions, two additional parameters are introduced: normal and tangential contact stiffness.

A simplified approach to simulating the impact of a mobile load was widely adopted, according to which a mobile moving load is represented by a group of time-varying concentrated forces on the trafficway line that ensure the "flow" of the load from node to node. This approach allows us to evaluate the dynamic performance of the span structure and requires minimal labor effort, but does not take into account the inertial characteristics of the moving load. The application of this technique in the suspension bridge design for mobile load is described in detail in [5].

This article studies the possibility of mixed simulation of load and span structure vibrations in Midas NFX finite element program using the built-in contact interaction mechanism based on the principle of resistance to interpenetration. At the first stage of the research, the mobile load is represented in the form of a three-dimensional body, the size of which are comparable with the beam cross section. The load is modeled by solid finite elements and has six inertial characteristics. The surface conditions of "surface-to-surface" type are used to determine the forces that prevent interpenetration. To verify the reliability of the results obtained, the method of separate mobile load modeling by use of several forces on the trafficway line is used [5].

\section{Simulation of joint vibrations of load and beam by means of contact conditions}

Contact analysis in the Midas NFX program provides ample opportunities in the area of modeling various types of interaction between two- and three-dimensional finite elements. In this paper, this mechanism is used to simulate the coupled vibrations of a moving load and a span structure.

The interaction of two bodies is represented by a system of "springs" at the contact boundary, the reaction of which is defined according to the condition of minimizing mutual penetration and is applied in the form of forces to the finite elements nodes. The Midas NFX program distinguishes between several types of contact: a node-to-surface, a surfaceto-surface, and a proper surface contact. The latter is realized in the case of large 
deformations of the structure, for example, in case of crush, and is not within the scope of this article.

When creating a design model, the master and slave interaction objects are assigned, that potentially can come into contact. Often, more accurate analysis results could be obtained by setting as the main object an object with greater stiffness or with a large grid. Plane bodies can participate in contact, as well as outer faces of solid finite elements. The grid of finite element nodes divides the objects into segments that participate in the determination of contact forces.

In the case of a node-to-surface contact (Figure 1), the algorithm checks the distance from the slave node to the master segment. If the slave node crosses the face of the master object, the repulsive force $F_{\mathrm{n}}$ begins to act on it and on the segment. For linear problems, the force $F_{\mathrm{n}}$ is determined by (1).

$$
F_{\mathrm{n}}=-\mathrm{k}_{\mathrm{n}} g_{\mathrm{n}}, \text { if } g_{\mathrm{n}}<0
$$

$\mathrm{k}_{\mathrm{n}}-$ contact stiffness; $g_{\mathrm{n}}-$ gap.

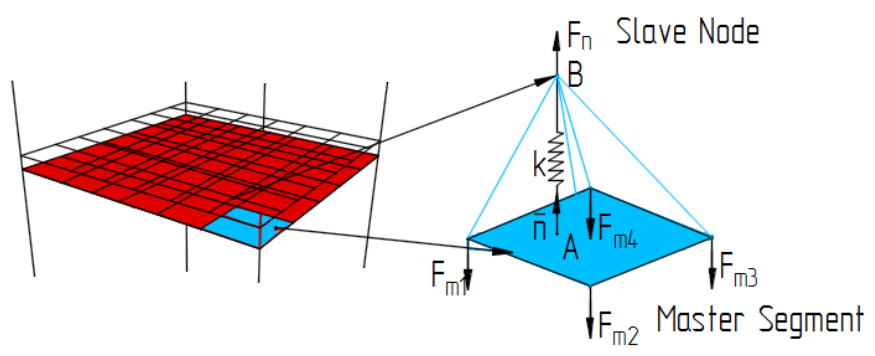

Fig. 1. Node-to-Surface Contact

After determining the force acting on the segment, the equivalent nodal forces are determined from the equilibrium equations $F_{\text {mi }}$.

In some cases, a node-to-surface contact can significantly reduce the accuracy of the results. During the modeling of coupled vibrations of an elastic-supported beam and a moving load, a surface-to-surface contact was used (Figure 2). In this case, the determination of the contact forces occurs in a similar manner, but the contact condition is checked for the i-th number of points of the slave segment, and then the nodal forces $F_{\mathrm{si}}$ and $F_{\mathrm{mi}}$ are found.

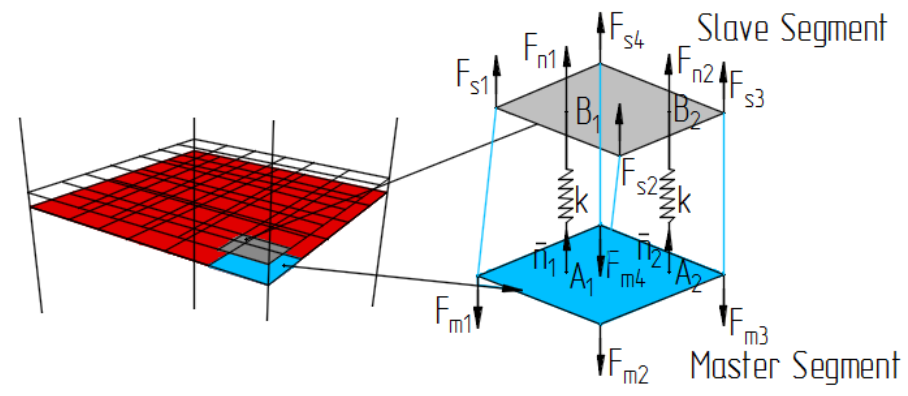

Fig. 2. Surface-to-Surface Contact

Since the contact rigidity varies sharply from 0 to $\mathrm{k}_{\mathrm{n}}$, depending on the size of the gap, in non-linear dynamic problems the problems with the convergence of the solution may arise. To avoid this, the Midas NFX uses modified contact forces, which are defined as follows: 


$$
\begin{array}{lr}
F_{\mathrm{n}}{ }^{\prime}=0 & \text { if } g_{\mathrm{n}}>\mathrm{d}_{1} \\
F_{\mathrm{n}}{ }^{\prime}=\frac{F_{\mathrm{n}}}{(\exp (1)-1)}\left[\left(\frac{-g_{\mathrm{n}}}{\mathrm{d}_{1}}+1\right)\left(\exp \left(\frac{-g_{\mathrm{n}}}{\mathrm{d}_{1}}+1\right)-1\right)\right] & \text { if } \mathrm{d}_{1}>g_{\mathrm{n}}>\mathrm{d}_{2} \\
F_{\mathrm{n}}{ }^{\prime}=\frac{F_{\mathrm{n}}}{(\exp (1)-1)}\left[\left(\frac{-\mathrm{d}_{\mathrm{n}}}{\mathrm{d}_{1}}+1\right)\left(\exp \left(\frac{-\mathrm{d}_{2}}{\mathrm{~d}_{1}}+1\right)-1\right)\right]+\mathrm{k}_{\mathrm{n}}\left(-g_{\mathrm{n}}-\mathrm{d}_{2}\right) & \text { if } g_{\mathrm{n}}<\mathrm{d}_{2}
\end{array}
$$

Thus, a nonlinear relationship is formed between the gap size and the contact force (Figure $3)$.

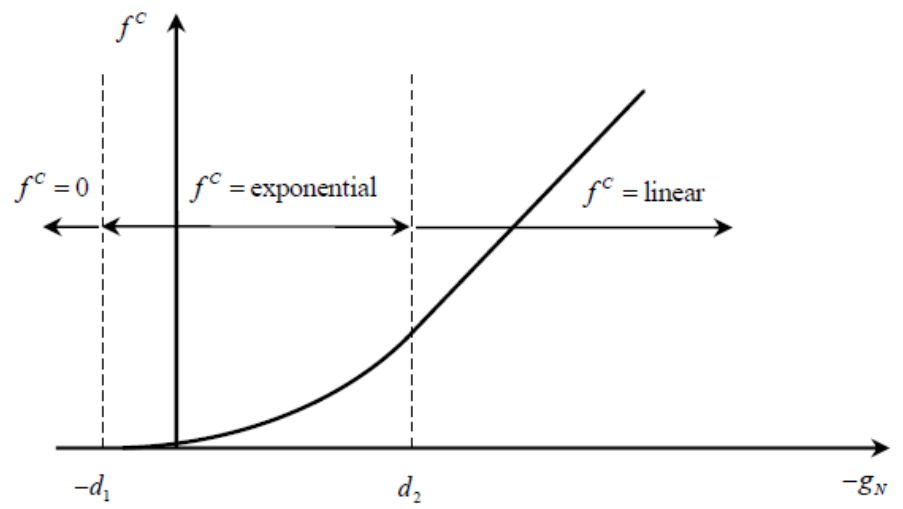

Fig. 3. Relationship between the gap and modified contact forces

Apart from the normal forces $F_{n}$, the tangential friction forces $F_{\tau}$ also arise on the contact boundary. Depending on the contact state, they are divided into static friction forces and sliding frictional forces. At this stage of calculations, their impact on the dynamic system was not taken into account.

\section{Description of the design scheme}

Consider the oscillations of elastically supported deformable beam with transition panels (Figure 4). Similar schemes are often used in the calculation of floating bridges [7].

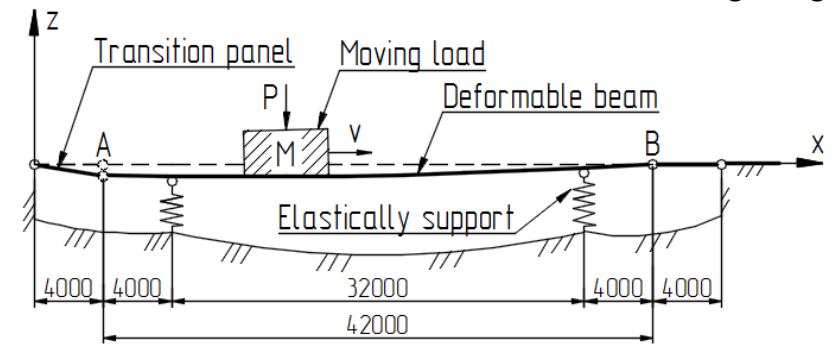

Fig. 4. Design scheme

The design scheme is a deformable beam with free ends, which is based on two elastic supports. It is assumed that a body of mass $\mathrm{M}$, to which force $\mathrm{P}$ is applied, moves along the dynamic system with a constant velocity. Smooth application of the load on the beam is provided by transition panels, pivotally connected at the ends. The stiffness of the beam along the length of the span structure is considered constant.

The program Midas NFX body of the beam was simulated solid 8-node finite elements (Figure 5). Elastic supports were set by FE "spring". To ensure the correct contact analysis 
procedure, the load begins to move from the start site, which is fixed from the displacements in space.

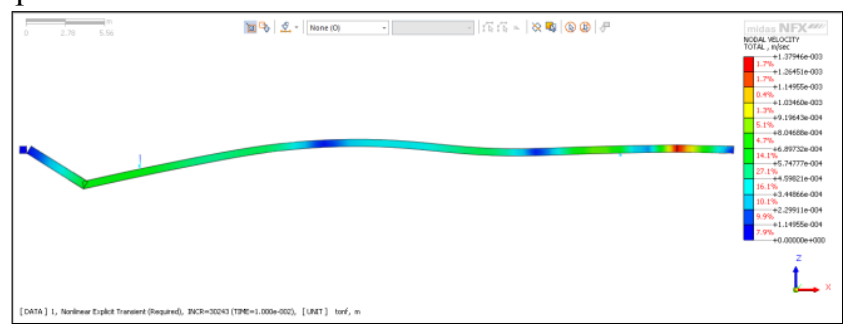

Fig. 5. Deformed view of the beam FEM (scale of deformation increased)

The moving load was modeled using surface-to-surface contact (6). The material of the beam and the load was assigned a specific density, which Midas NFX is using for calculation of inertial characteristics. Own weight of structures was not taken into account in the calculation. The motion of the load on the beam was provided by the kinematic boundary conditions $\mathrm{V}$. To perform calculations, an explicit method of central differences was used.

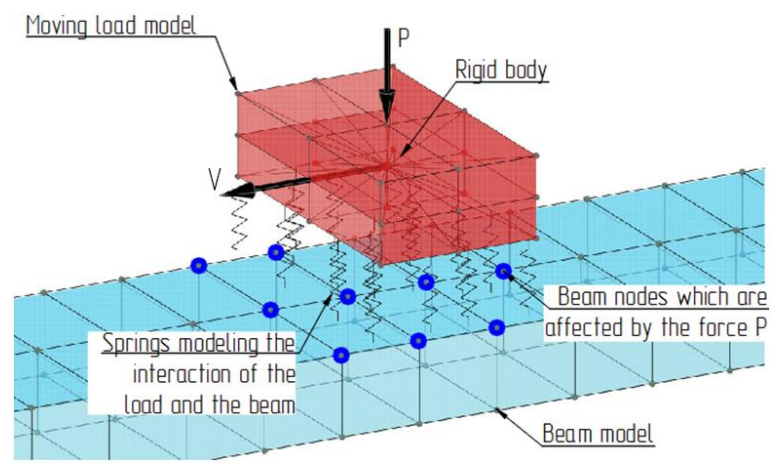

Fig. 6. Moving load model

To verify the reliability of the results, the method of separate modeling of the moving load and beam vibrations proposed by authors in [5] was used. According to this technique, the fluctuations occur under the influence of a group of forces on the line of the road. To perform the calculations, a flat rod finite element model of the beam is used (Figure 7). The moving load is represented as:

$$
F_{\mathrm{i}}(t)=P(t) f_{\mathrm{i}}(t)
$$

$F_{\mathrm{i}}(t)$ - concentrated force in the $\mathrm{i}$-th node

$f_{\mathrm{i}}(t)$ - an auxiliary function that sets what percentage of the load comes to the $\mathrm{i}$-th node.

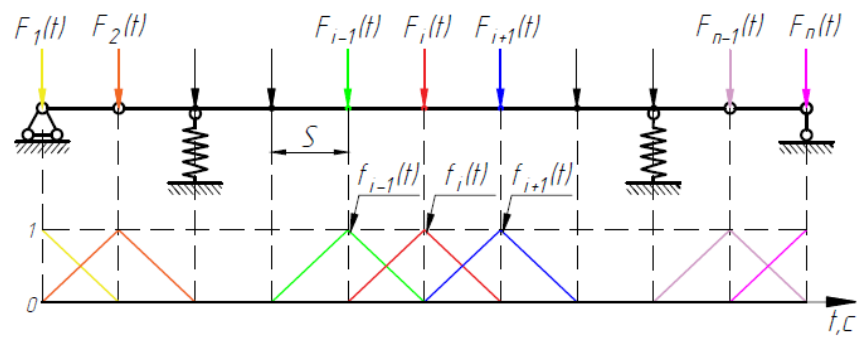

Fig. 7. The application schema group of forces 
The method of superposition of modes was used to analyze the oscillations of the rod system. When solving the problem on generalized eigenvalues, the iteration method in the subspace is chosen.

\section{Numerical investigation}

Using the two approaches described above, a series of numerical experiments was performed on a simplified model of elastically supported deformable beam with transition panels. The characteristics of the dynamic system in the calculations are taken as follows: $\mathrm{m}=0.888 \mathrm{~T} / \mathrm{m}$ - linear mass of the beam; EI $=6.48 \mathrm{e} 7 \mathrm{Tm}^{2}-$ bending stiffness of the beam; $\mathrm{c}_{1}=\mathrm{c}_{2}=120 \mathrm{Tc} / \mathrm{m}-$ stiffness of elastic supports.

For the analysis of the oscillatory process, the displacements of the beginning (point A) and the end (point B) of the beam were investigated. Figure 8 shows the vertical movements of the left free end of the beam when a non-inert load moves. The calculation was performed using two methods. The results obtained suggest that the accepted calculation schemes can be considered equivalent.
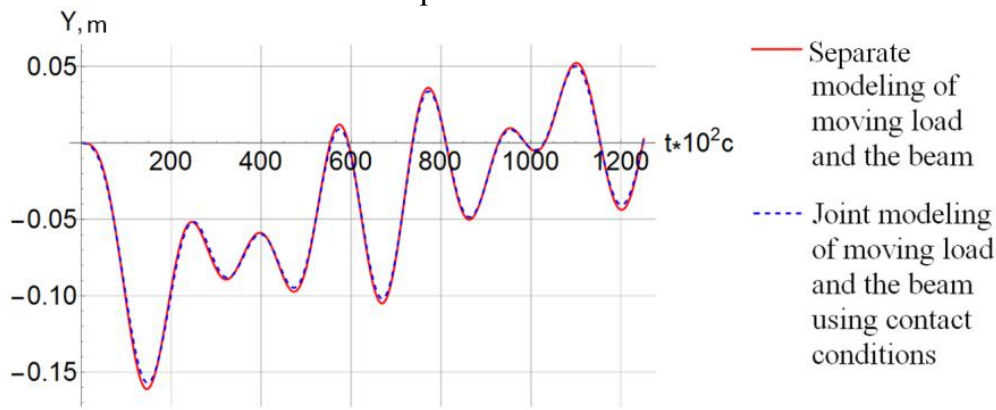

Fig. 8. Vertical displacements of point A. Two approaches for moving load modeling

Figure 9 shows the results of the dynamic system calculation by the method of separate modeling. During the calculations, the step of application of nodal forces modeling the moving load was varied. According to the data obtained, it can be concluded that the step $\mathrm{S}=1.25 \mathrm{~m}$ for this dynamic system is optimal in terms of calculation time and calculation accuracy. At the next stage, the influence of the mass characteristics of the load on the characteristics of the dynamic system oscillations was investigated (Figure 10). Analyzing the graphs, we can conclude that with the increase in the mass of the moving load, the period and amplitude of the oscillations of the elastically supported dynamic system increases.

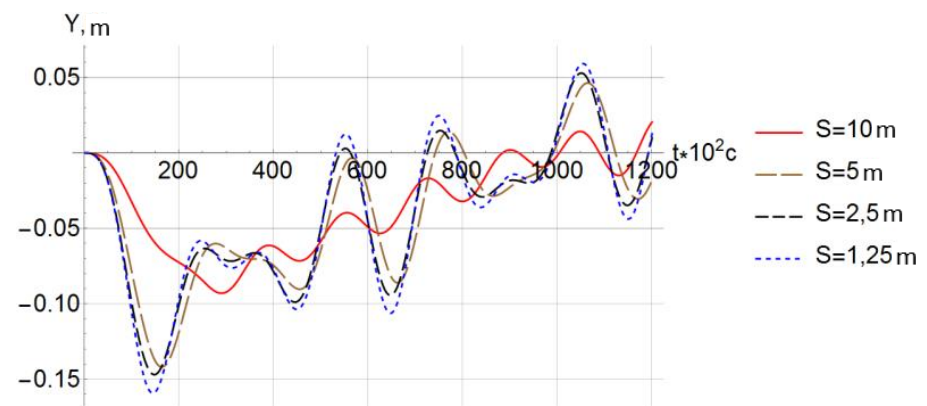

Fig. 9. Vertical displacements of point A. Separate modeling of moving load and the beam. 
It should be noted that the calculation of this elastically supported dynamic system should be performed taking into account the inertial parameters of the mobile load. When moving a load weighing 10 tons, the maximum vibration amplitude increases by $18 \%$.

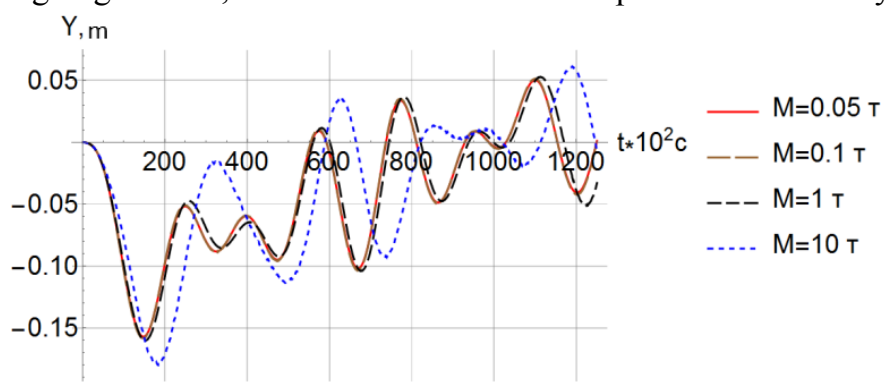

Fig. 10. Vertical displacements of point A. Joint modeling of moving load and the beam.

An important parameter in the joint modeling of the load and beam vibrations is the contact stiffness. Its value can be adjusted by the user using the scale factor. This parameter allows to increase or decrease the spring stiffness at the contact boundary by a specified number of times. Figure 11 shows graphs of vertical movements of point A, with different values of the scale factor.

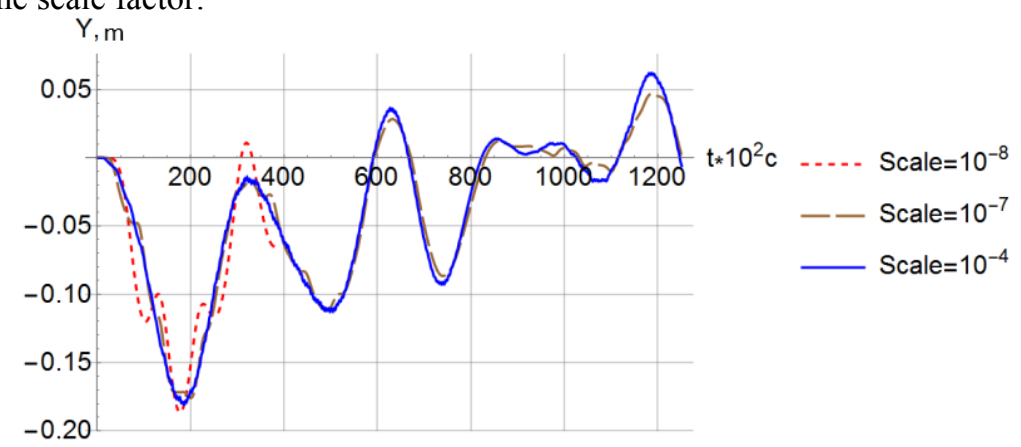

Fig. 11. Vertical displacements of point A. Joint modeling of moving load and the beam.

A significant reduction in contact stiffness leads to the fact that the load begins to fluctuate, moving along the surface of the beam, which is clearly visible on the graph. At low values of contact stiffness (Scale $=10^{-8}$ ), the forces preventing interpenetration may be insufficient to continue to hold the load on the contact surface. As a result, the moving load model can fall through the beam body, making further calculations meaningless.

\section{Summary}

Using the contact interaction mechanism presented in the Midas NFX, an analysis of the vibrations of an elastic-supported dynamic system along which the simplified model of the vehicle is moving is made. The obtained results testify to a range of advantages of the given method of mobile load modeling.

First, it allows us to take into account the inertial characteristics of the mobile load, which under certain parameters of the dynamic system could significantly affect the nature of the vibrations of the span structure. Secondly, the algorithm used can be applied to describe more complex models of vehicles with many degrees of freedom, which is the goal of further research. Thirdly, the described methodology does not require the use of additional software to determine the contact forces. The Midas NFX calculating model 
contains all information about the model of the mobile load and the beam, considering their vibrations in the aggregate. Thus, the application of surface conditions while using modern finite element analysis complexes for the dynamic systems design for mobile load is an actual and promising line of research.

\section{References}

1. Yu. Arkhipenko, diss. cand. tech. sciences: 05.23.11. Moscow, 177 (2006)

2. E. Krugovova, G. Mikheev, R. Kovalev, B. Bryansk st. tech. Un., 9, 39-48 (2010)

3. I. Alferov, diss. cand. tech. sciences: 05.23.17. Moscow, 115 (2015)

4. V. Zylev, SPC Engineer, 144 (1999)

5. G. Kadisov, V. Chernyshov, Eng. Con. J.: SPbPU., 8, 56-63 (2013)

6. S. Gridnev, Privolzhsky Sc. J., 7, 64-71 (2008) 